\title{
Treatment Strategies Against Triple-Negative Breast Cancer: An Updated Review
}

\author{
Mudasir Maqbool' \\ Firomsa Bekele $\mathbb{I D}^{2}$ \\ Ginenus Fekadu (iD ${ }^{3,4}$ \\ 'Department of Pharmaceutical Sciences, \\ University of Kashmir, Srinagar, Jammu \\ and Kashmir, India; ${ }^{2}$ Department of \\ Pharmacy, College of Health Sciences, \\ Mettu University, Mettu, Ethiopia; \\ ${ }^{3}$ School of Pharmacy, Faculty of Medicine, \\ The Chinese University of Hong Kong, \\ Shatin, NT, Hong Kong; ${ }^{4}$ Department of \\ Pharmacy, Institute of Health Sciences, \\ Wollega University, Nekemte, Ethiopia
}

Correspondence: Firomsa Bekele Department of Pharmacy, College of Health Science, Mettu University, Mettu, Ethiopia

Email firomsabekele2I@gmail.com

\begin{abstract}
Triple-negative breast cancer (TNBC) is associated with an increased risk of early recurrence and distant metastasis, as well as the development of therapeutic resistance and poor prognosis. TNBC is characterized by a wide range of genetic, immunophenotypic, morphological, and clinical features. TNBC is coined to describe cancers that lack estrogen receptor (ER), progesterone receptor (PR), and human epidermal growth factor receptor 2 (HER2). As a result, hormonal or trastuzumab-based treatments are ineffective in TNBC patients. TNBCs are biologically aggressive, and despite some evidence that they respond to treatment better than other forms of breast cancer, the prognosis remains poor. This is attributed to a shorter disease-free interval in adjuvant and neoadjuvant settings, as well as a more aggressive metastatic course. TNBC has a lot of clinical ramifications. In terms of new treatment methods, TNBC has lagged behind other types of breast cancer. There are not many options for treating this form of breast cancer because it is progressive. Many effective treatments for most breast cancers block the growth-stimulating effects of ER, PR, and/or HER2, leaving TNBC with few choices. Finding new and effective treatment options for TNBC remains a critical clinical need. To develop more effective drugs, new experimental approaches must be tested in patients with TNBC.
\end{abstract}

Keywords: breast cancer, progesterone receptor, genetic, human epidermal growth factor receptor 2

\section{Introduction}

Breast cancer is one of the most common cancers among women worldwide. It has different pathological and molecular subtypes, and different therapies with different clinical results are needed to treat it. ${ }^{1}$ Numerous targeted drugs are being tested in preclinical and clinical studies to combat the complications of breast cancer. Since the late $1990 \mathrm{~s}$, mortality rates have decreased due to early detection using palpation and mammograms, as well as adjuvant systemic therapy for treatment. However, some breast tumors remain resistant to traditional therapies, which may be due to the activation of pro-proliferative, anti-apoptotic, and pro-survival signaling pathways. These factors work together to cause drug resistance, which affects the clinical conditions' vulnerability to monotherapy. Apart from the urgent problem of drug resistance, which is on the verge of becoming a medical epidemic, current medications are often known to cause such adverse effects, which have a direct impact on the treatment outcome. $^{2,3}$ As a result, there is a strong desire to develop new therapeutic methods to effectively treat this disorder. TNBC is a biologically active form of breast cancer that accounts for approximately $15-20 \%$ of all breast cancer cases. Estrogen receptors (ERs), progesterone receptors (PRs), and human epidermal growth factor receptor 2 
(HER2) are absent. ${ }^{4}$ TNBC has a wide range of genetic, immunophenotypic, morphological, and clinical characteristics. TNBCs are typically greater in size and heterogeneous in nature than other breast cancer subtypes. TNBC affects the lymph nodes. TNBC can be divided into six subtypes, as per new research, each with its own gene expression profiles and ontologies. ${ }^{5}$ The lack of ERs, PRs, HER2 expression, and predictors of resistance to standard chemotherapy make designing specific TNBC drug therapies difficult. ${ }^{6}$

Androgen receptors (ARs) have been shown to play an important role in TNBC cell growth, invasion, migration, and apoptosis, both of which contribute to disease complications. Targeting ARs with AR inhibitors may be the most effective therapy for TNBC. ${ }^{7}$ Different inflammatory molecules, such as tumor-associated macrophages (TAMs), tumor infiltrating lymphocytes (TILs), and cytokines, suppress the immune response to TNBC during chemotherapy, increasing the risk of TNBC complications. ${ }^{7}$ In patients with TNBC, therapies targeting these inflammatory molecules can improve disease-free and overall survival rates. In TNBC, miRNAs play an important role in cancer cell survival, proliferation, invasion, and metastasis, and manipulating these miRNAs can help reduce the risk of early relapse and metastasis. ${ }^{8}$ Furthermore, although TNBC lacks estrogen receptor (ER) expression, the presence of estrogen receptor dependent receptors (ERRs) is linked to poor clinical outcomes in TNBC patients. ${ }^{9}$ Similarly, autophagy inducers, nuclear factor kappa $\mathrm{B}(\mathrm{NF}-\kappa \mathrm{B})$, and mammalian target of rapamycin (mTOR) have been found to play a role in disease progression. ${ }^{10,11}$ The use of inhibitors to target these molecules may be a reliable treatment technique for TNBC. To develop precise and reliable agents that can effectively target TNBC, it is critical to elucidate the prognostic markers of TNBC. TNBC prognostic factors include node status, cathepsin-D, Ki67 index, BRCA1 promoter methylation value, and p53. ${ }^{12,13}$ These prognostic tools are usually effective in predicting disease-free survival and overall survival rates in TNBC patients, and in cases of poor prognosis, other better treatment options may be considered (Figure 1).

\section{Treatment Strategies for Triple Negative Breast Cancer}

TNBCs are a heterogeneous and aggressive form of cancer, for which there are no scientifically validated biologically targeted effective treatments. The lack of ERs, PRs, and HER2 makes finding a reliable treatment alternative for TNBC extremely difficult. In the following sections, we will concentrate on some of the most successful and

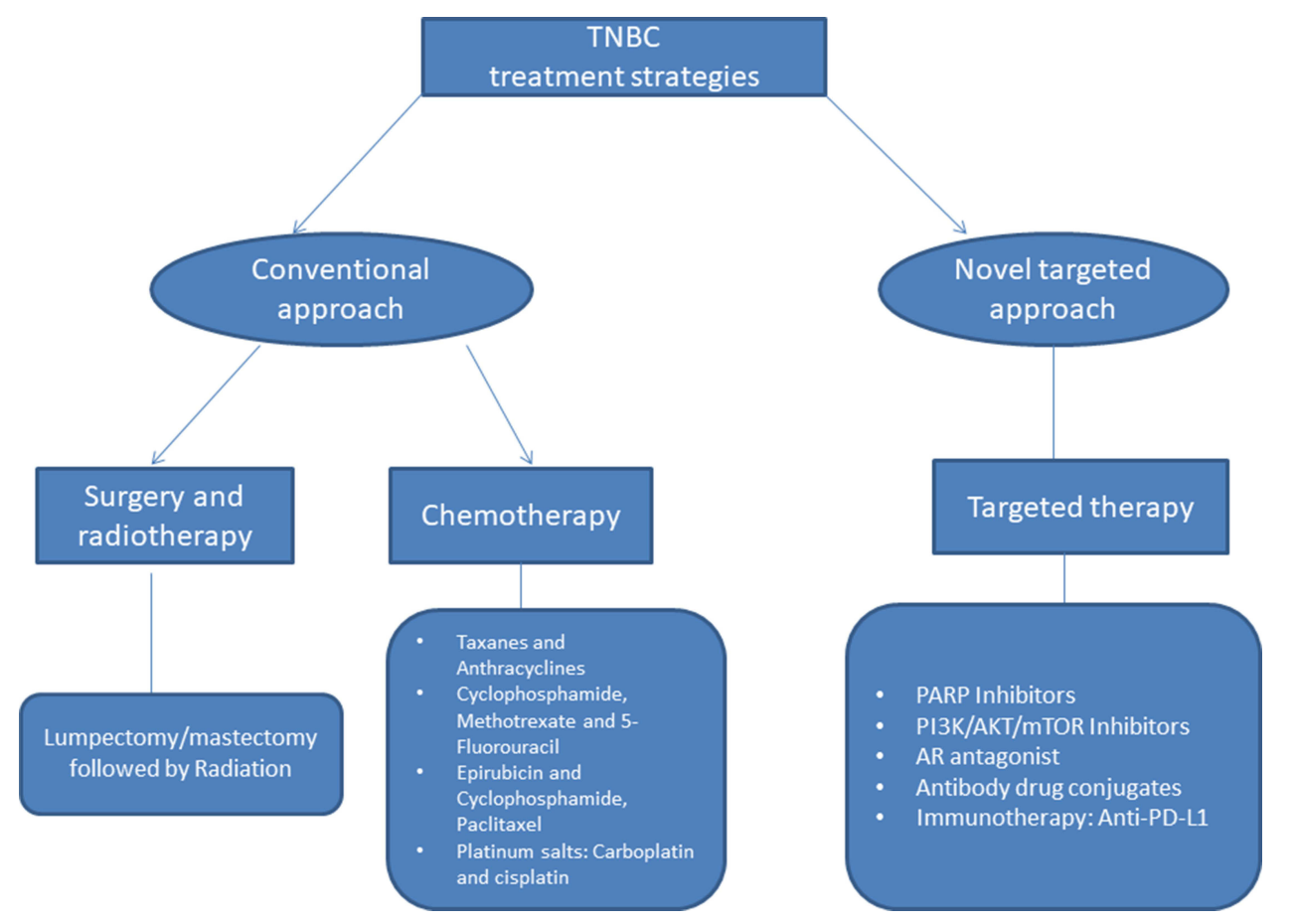

Figure I Summarized conventional and novel targeted approach to treat triple-negative breast cancer.

Abbreviations: AR, androgen receptor; PARP, poly (ADP-ribose) polymerase; PD-LI, Programmed death ligand I; PI3K/AKT/mTOR, Phosphatidylinositol-3-kinase (PI3K)/ AKT and the mammalian target of rapamycin (mTOR). 
dependable treatment solutions for TNBC, as well as the reasons for their failure.

\section{Targeting Androgen Receptors}

In a subset of TNBC, the role of androgen signaling begins to emerge. ARs, a form of steroid hormone receptor, have recently been discovered to be prognostic and treatment predictive markers in patients with TNBC. AR is found in approximately $80 \%$ of invasive breast cancers and approximately $30 \%$ of TNBC patients. ${ }^{14}$ The levels of AR expression in TNBCs varied greatly. Patients with ARdependent TNBC have a better prognosis than those with AR-independent TNBC. As a result, agents targeting ARs may be the most effective treatment marker for TNBC. According to a study conducted by Bonnefoi et al, antiandrogen drugs may be a reliable treatment marker for TNBC. For this review, 146 patients from 27 different centers were selected. An appropriate tissue sample was available for 136 of 146 patients, and they were AR positive and triple negative. They examined the effectiveness and safety of abiraterone acetate (AA) plus prednisone in women with AR+ and ER-, PR-, HER-2metastatic or inoperable locally advanced breast cancer in a multicenter single-arm step second study. At the end of six months, the clinical benefit rate was $20 \%$. This suggests that AA plus prednisone is effective for ARdependent TNBC patients, and it can be inferred from this study that targeting ARs may be a potential treatment strategy for TNBC. ${ }^{15}$

Androgen-targeting has shown promising early results and should be further studied in the right TNBC patients. AR IHC (Androgen receptor immunohistochemistry) expression (with varied cutoffs) has been used as a selection criterion in therapeutic trials including AR targeting. On the other hand, in preclinical studies of this treatment strategy, AR IHC and gene expression-defined subtypes were used (luminal or LAR subtypes). Due to the complexity of interconnected signaling pathways, IHC positive for AR alone is unlikely to reliably identify individuals who will benefit from AR modifying medicines. ${ }^{16-18}$ Subtypes of tumour AR dependence have emerged in recent years based on gene expression (LAR, intrinsic luminal subtype, PREDICT AR subtype). ${ }^{6,18-21}$ Prospective studies are needed before they may be transferred into routine clinical practice. Additional translational research should also focus on the changes in tumour AR dependency under the stresses of routine chemotherapy and whether primary or metastatic tumour androgen dependency is most likely to correspond to antiandrogen treatment response in the main tumour. ${ }^{18,21}$

Efficacy of single-agent AR inhibitors in clinical trials was shown to be limited. AR inhibitors can now be tested in a new generation of clinical studies, thanks to recent breakthroughs in our knowledge of androgenic signaling in TNBC. A solid set of biomarkers for the detection of TNBC cancers that are androgen dependent must be established at the same time as novel AR-targeting drugs are produced and tested in clinical trials. Androgen signaling has a multifaceted role, but it emerges as a therapeutic target for a subset of patients with this aggressive disease that lacks molecular targets. Antiandrogens are well tolerated and have known and acceptable safety profiles. It is being tested in clinical trials as a single drug or in combination with other antiandrogen medicines and cytotoxic chemicals. In the future, standardized and reproducible methods for identifying AR-dependent malignancies will allow researchers to adapt clinical trials to the correct patient population and allow doctors to better predict patients' responses in future clinical trials. ${ }^{18}$

ARs play a role in TNBC by inducing cell proliferation, invasion, and metastasis, as well as inhibiting apoptosis. The following subsections provide a quick overview of how ARs play a role in complicating TNBC and a potential treatment plan.

\section{Role of Androgen Receptors in Inducing Cell Proliferation and Inhibition of Apoptosis in Mesenchymal Stem Like}

TNBC has a subtype called mesenchymal stem-like (MSL). AR was found to be strongly expressed in the MSL subtype of TNBC. In vitro and in vivo studies performed by Zhu et al showed the tumorigenic function of ARs and the inhibitory effect of bicalutamide in AR+ MSL TNBC. The effect of AR on cell viability and apoptosis in the MSL TNBC MDA-MB-231 cell line was investigated using cell counting, flow cytometry, the MTT assay, and Western blotting to examine the protein expression of p53, p73, p21, and cyclin D1. Using a ChIP assay, AR binding to p73 and p21 was investigated. MDAMB-231 cells were then implanted into the naked mice. Tumor growth was examined after DHT or bicalutamide treatment, and the expression of AR, p73, and p21 was determined using immunohistochemistry. The results showed that the AR agonist DHT stimulated cell proliferation and inhibited apoptosis in MSL TNBC cells. AR improves the viability of MDA-MB-231 xenografts 
in vivo Bicalutamide, on the other hand, is an AR antagonist that induces apoptosis and inhibits the growth of MSL TNBC MDA-MB-231 cells. ${ }^{22}$ This suggests that AR plays a significant role in TNBC development. As a result, AR inhibitors can be used as dependable treatment markers for TNBC.

\section{Role of Androgen Receptors in Inducing Invasion and Migration}

Androgen receptors (ARs) have been discovered to play a role in the development, invasion, and metastasis of TNBC. Caiazza et al explored whether targeting AR might be a hormonal approach for treating TNBC. The anti-androgen drug enzalutamide was selected for the analysis. The results showed that enzalutamide could successfully inhibit AR-induced cell growth, invasion, and metastasis in TNBC. Enzalutamide inhibits AR-induced cell proliferation, invasion, and metastasis by inhibiting the transcription factors AP-1 and SP-1. In an ARdependent manner, the first-generation anti-androgen flutamide was also found to be efficient in inhibiting cell development, invasion, and metastasis. The effect of AR on TNBC was studied in 2091 patients, and it was discovered that there is a significant link between AR expression and poor overall survival, leading to the conclusion that AR complicates TNBC by inducing cell development, invasion, and metastasis. ${ }^{23}$ Thus, it can be inferred that AR plays a major role in the violent nature of TNBC. However, this finding raises the possibility that antiandrogen drugs may be a useful hormonal tool in the treatment of TNBC. As a result, developing more anti-androgen drugs to tackle the problem of TNBCs has become a priority.

\section{Targeting Inflammatory Molecules}

Chemotherapy performance in TNBC is also influenced by the immune response created against the tumor cells that die during treatment. Genetic and epigenetic modifications of TNBC aid tumor-associated antigens in developing resistance to the immune system response. Various pathways, such as immune checkpoints, which are involved in mediating immune tolerance and mitigating collateral tissue damage, aid tumor cells in gaining resistance to chemotherapy. The involvement of inflammatory molecules, such as macrophages, cytokines, chemokines, and tumor infiltrating lymphocytes (TILs) has been shown to influence overall survival rates in TNBC in several studies. The involvement of activated inflammatory molecules in the tumor microenvironment inhibits the immune response against tumor cells, thereby increasing TNBC complications. ${ }^{24}$ As a result, increasing disease-free survival rates in TNBC requires a focus on these inflammatory molecules. To find a solution for TNBC, various therapies targeting these inflammatory molecules must be investigated. The role of various inflammatory molecules in complicating TNBC is discussed in this section.

\section{Role of Tumor Infiltrating Lymphocytes (TIL)}

The existence of activated inflammatory molecules also leads to its aggressive nature, according to various studies. TILs, a form of inflammatory molecule, have been linked to TNBC complications in several studies. High TIL levels are linked to a higher overall survival rate in patients with TNBC. TIL levels can be used as predictive and prognostic markers to assess the effectiveness of different standard therapies, such as neoadjuvant and adjuvant chemotherapy. Targeting inflammatory molecules may be the most effective way to combat TNBC. Recent research has shown that immune modulatory agents, such as immune checkpoint inhibitors, would have a promising effect against TNBC. Another study by Teijido et al supports the idea that targeting molecules expressed on the surface of TNBC may be a more effective treatment approach. T-cells within the tumor setting activate programmed cell death protein 1 (PD-1) and its ligand (PDL1), which is known to suppress the local immune response. In TNBC, PD-1-PD-L1 is essential for sustaining immunosuppression in the tumor setting. During clinical trials, Garcia Teijido et al discovered that targeting PD-1 with the inhibitor pembrolizumab and PD-L1 with the inhibitor atezolizumab showed promising results against TNBC. ${ }^{25,26}$ As a result, PD1-PDL1 is currently being considered as a potential therapeutic target for TNBC.

\section{Role of TNF-Alpha}

While the mechanism by which inflammatory molecules mediate TNBC progression is still unknown, there is a growing body of evidence suggesting that inflammatory molecules play a role in TNBC complications. Another inflammatory molecule, $\mathrm{TNF}-\alpha$, plays a role in disease progression, according to a study conducted by Qiao et al. They discovered that transcription factor AP-1 aids in the development of cancer caused by inflammation rather than mediating the inflammatory response, and they used chIP-seq to show that the cistrome for AP-1 transcription factor c-Jun is made up of 13,800 TNF-alpha 
transcriptome-binding regions. Approximately one-third of the TNF- $\alpha$ transcriptome is controlled by c-Jun. In TNBCs, high levels of c-Jun expression have been linked to poor clinical outcomes. They discovered that TNF-alpha expression mediated by c-Jun increases the malignant characteristics of TNBC. They also mentioned that NF$\kappa \mathrm{B}$ may be a key transcription factor in the regulation of TNF-alpha, while c-Jun has little effect on TNF-alpha in the mediation of TNBC malignancy. ${ }^{27-33}$ As a result, TNF- $\alpha$ is implicated in complications of TNBC, and therapies targeting TNF- $\alpha$ could be a more effective treatment option for TNBC.

\section{Role of Tumor Associated Macrophages and Cytokines}

To learn more about the etiology of TNBC and develop drugs to treat it, researchers must identify all the factors that contribute to the disease's complications. Wang et al investigated the expression profile of tumor-associated macrophages (TAMs) and cytokines, as well as their functions in TNBC complications. RT-PCR and ELISA were used to check the expression of cytokines such as IL6, IL10, IL-1B, IL-12, chemokine, and macrophage inflammatory protein -2 (MIP-2) in 48 TNBC cases. TAMs were also detected using immunohistochemical S-P staining. In TNBC patients after surgery, 34 out of 48 were found to be CD68 positive, and IL-6, CCL-5, and even TAM expression levels were all upregulated. The presence of both factors leads to a poor prognosis in TNBC. The development of drugs targeting these molecules may be a ray of hope in the battle against TNBC. ${ }^{34}$ Based on the evidence presented above, inflammatory molecules play a role in complicating the disease and contributing to TNBC's aggressive behavior. Therefore, the development of drugs targeting these molecules may be a valuable therapeutic strategy for TNBC. The mechanisms underlying these facts must also be explored in depth, as this will aid the scientific community in developing targeted TNBC drug therapy.

\section{Targeting MicroRNAs}

It has been challenging to establish new and effective therapies to improve TNBC patients' overall survival rates due to the heterogeneity of TNBC and the lack of frequency drive mutations other than TP53. MicroRNAs have been shown to play a role in TNBC cancer cell survival, proliferation, invasion, and metastasis, and miRNAs that target these miRNAs can reduce the risk of relapse and metastasis. MicroRNAs (miRNAs) are global regulators of cancer cell survival, proliferation, and migration. $^{34}$ Therefore, miRNAs may be an alternative treatment for TNBC. In this segment, we will examine how miRNAs regulate cell proliferation, invasion, and metastasis in TNBC.

\section{Role of MicroRNAs in Inducing Invasion and Metastasis}

The molecular basis for metastasis and disease relapse in patients with TNBC remains largely unknown. The complications of TNBC are exacerbated by metastasis and early relapse. Yan et al investigated the function of miRNAs in inducing invasion and metastasis in TNBC. They discovered that miR-136 functions as an antiinvasive microRNA in TNBC and inhibits invasion and metastasis in the mesenchymal subtype of TNBC in their research. They discovered that anti-invasive and antimetastatic miR-136 were downregulated in TNBC. Overexpression of RASAL2 in TNBC prevents cell migration and invasion mediated by miR-136 As a result, it can be concluded that miR-136/RASAL2 serves as a suppressor of TNBC invasion and metastasis and that it can be successfully used as a drug therapy to treat the complications of TNBC invasion and metastasis. ${ }^{8}$

\section{Role of microRNAs in Inducing Cell Proliferation}

MicroRNAs have been found to play a role in cell proliferation in TNBC. Phan et al discovered that overexpression of miR-1296 in TNBC cells prevents cell proliferation and causes apoptosis. Overexpression of miR-1296 also resulted in a significant reduction in the $S$ phase of the cell cycle. Researchers have used miRNA pRT-PCR, colony formation assays, cell cycle analysis, and apoptosis assays to investigate this. Other methods, such as immunoblotting and luciferase assays, were used to determine the expression of miRNA-1296 target protein. Based on their results, Phan et al concluded that miR-1296 targets cyclin D1 (CCND1), a cell cycle protein. The transcription factor miR1296, when overexpressed in TNBC cells, suppresses the expression of CCND1. Overexpression of MiR-1296 can be counteracted by CCND1 overexpression. Consequently, there is a negative relationship between miRNA-1296 and CCND1. ${ }^{35}$ Furthermore, miRNAs can be used to treat TNBC as an effective therapeutic strategy. However, more research is needed to fully understand the potential of miRNAs and their use as a treatment strategy for TNBC. 


\section{Targeting Estrogen Receptor Related Receptors}

Several studies have discovered the role of estrogen receptor associated receptors (ERRs) in TNBC complications. In patients with TNBC, high levels of ERR alpha are associated with a poor prognosis. YM et al discovered that inhibiting ERR alpha with the inverse agonist XCT790 suppressed cell proliferation and induced mitochondrial-dependent apoptosis in TNBC cells in their study. XCT-790 suppressed cell proliferation by increasing the expression of $\mathrm{p} 53$ and $\mathrm{p} 21$ (growth inhibitory proteins). XCT-790 increases the levels of ATF4/6, XBT-1, and CHOP, both of which are proteins associated with ER stress. The presence of reactive oxygen species (ROS) in TNBC increases when SOD1/2 is inhibited by XCT-790. On the other hand, the ROS scavenger NAC prevented ER stress and growth arrest caused by XCT-790. ERK1/2, JNK, p38-MAPK, Akt, NF-kBp65, and IB can all be effectively targeted by XCT-790. Other ERK1/2, JNK, Akt, and NF-B inhibitors also prevent TNBC from developing ROS in response to XCT-790. Based on these results, it can be concluded that XCT-790 treatment causes activation of Akt, ERK, NFB, and p38-MAPK-based ROS in TNBC cells. Validation of these results was performed in vivo on MDA-MB-231 xenograft tumors. Treatment with XCT-790 inhibits cell growth in MDA-MB-231 xenograft tumors, which is linked to increased expression of p53, p21, ER-stress related proteins, and Bcl2 downregulation. ${ }^{9}$ All of this research supports the notion that ERRs play a role in the progression of TNBC, and that using anti-ERRs as a therapeutic option for TNBC will prove to be a successful drug candidate. Furthermore, it lays the groundwork for the potential production of drugs that target ERRs to combat TNBC complications.

\section{Targeting Mammalian Target of Rapamycin}

The downstream regulator of phosphatidylinositol 3-kinase (I)3K], the mammalian target of rapamycin (mTOR), is one of the most well-known signaling pathways involved in cancer complications. In TNBC, the $\mathrm{P}(\mathrm{I}) 3 \mathrm{~K}-\mathrm{mTOR}$ pathway was found to be activated at the genome, gene expression, and protein levels. mTOR complexes mTORC1 and mTORC2 are known to exist. TNBC is difficult to treat due to mTOR activation at various levels in tumors. The development of mTOR inhibitors could be a successful treatment strategy for TNBC. Zhang et al investigated the effects of two
mTOR inhibitors, sirolimus (rapamycin) and temsirolimus (CCI-779), on patient-derived xenografts with several TNBC subtypes. ${ }^{36}$ They observed $77-99 \%$ growth inhibition in patient xenografts after drug testing with mTOR inhibitors. However, they also mentioned that no tumor was completely eradicated; rather, the activation of the mTOR pathway was reduced. These results clearly show that mTOR inhibitors are successful against TNBC, but that they need to be used in combination with other treatments to fully eliminate the complications. In their study, Zhang and colleagues discovered that mTOR inhibitors have a cytostatic effect on tumor development, but that they are insufficient to completely eradicate tumor mass. Additional drugs, in addition to mTOR inhibitors, are necessary to completely shrink the tumor. New drug formulations containing mTOR catalytic inhibitors, dual kinase inhibitors of mTOR and P(I) 3 $\mathrm{K}$, and combined targeting of selective allosteric pan-Akt inhibitor MK-2206 with mTOR inhibition are currently being studied in order to fully eradicate TNBC. ${ }^{37}$ To combat TNBC, researchers should concentrate on developing combinatorial drug therapies that include mTOR inhibitors and other medications.

\section{Targeting Nuclear Factor Kappa B}

Nuclear factor kappa B (NF- $\mathrm{kB})$ is a transcription factor involved in immune control, inflammation, and tumorigenesis in several cancers. When IKKB kinase is stimulated in response to proinflammatory and prooxidative stimuli, it phosphorylates and degrades I $\mathrm{KB}$ proteins. ${ }^{38,39}$ Another study investigated the impact of lapatinib on NF- $\mathrm{KB}$ activation in TNBC. Lapatinib is a tyrosine kinase inhibitor that inhibits both the EGRR and HER2 receptors. They examined how lapatinib and proteasome inhibitors affected TNBC. Chen and colleagues concluded that lapatinib inhibited NF- $\mathrm{KB}$ activation in TNBC, independent of EGFR/HER2 inhibition, using immunoprecipitation assays, immunoblotting, RT-qPCR, and luciferase assays. There were no other EGFR inhibitors that synergized with proteasome inhibitors, except for lapatinib. According to these findings, treatment of TNBC with lapatinib can increase oncogene addiction to $\mathrm{NF}-\kappa \mathrm{B}$, thereby increasing the anti-tumor activity of proteasome inhibitors. ${ }^{40}$ These results strongly suggest that combination therapy combining proteasome inhibitors and lapatinib may be a viable treatment option for TNBC. 


\section{Targeting Autophagy}

Autophagy is a biological process in which the cells of the body are killed. Autophagy has been linked to TNBC complications in a variety of studies. According to Liang et al, CQ, an autophagy blocker, effectively targets cancer stem cells (CSCs) in TNBC by inhibiting autophagy,

Table I Therapeutic Strategies and Targeted Agents That May Be Used in Specific Subtypes of Triple Negative Breast Cancer

\begin{tabular}{|c|c|c|}
\hline TNBC Subtype & Therapeutic Strategies & Therapeutic Targeted Drugs \\
\hline $\begin{array}{l}\text { LAR (luminal } \\
\text { androgen receptor) }\end{array}$ & $\begin{array}{l}\text { Inhibit FOXAI, AR signaling, and } \\
\text { ERBB4 signaling }\end{array}$ & $\begin{array}{l}\text { PI3K inhibitors Eg, Idelalisib } \\
\text { mTOR inhibitors Eg, RapaLink-I, rapamycin, everolimus, } \\
\text { Nonsteroidal antiandrogens Eg, bicalutamide }\end{array}$ \\
\hline $\begin{array}{l}\text { MSL (mesenchymal } \\
\text { stem-like) }\end{array}$ & $\begin{array}{l}\text { Inhibit, PI3K, mTOR, EMT, Wnt, } \\
\text { TGF } \beta \text {, MAPK, Rac, Scr, PDGF }\end{array}$ & $\begin{array}{l}\text { Scr inhibitors Eg, Dasatinib, Bosutinib } \\
\text { MAPK inhibitors Eg, Dabrafenib, Trametinib } \\
\text { PI3K inhibitors Eg, Idelalisib } \\
\text { mTOR inhibitors Eg, RapaLink-I, rapamycin, everolimus } \\
\text { Growth Factor inhibitors Eg, Vandetanib, Bevacizumab, Pertuzumab, } \\
\text { Ramucirumab, Trastuzumab, Axitinib, Cabozantinib, Ceritinib, Erlotinib, Gefitinib, } \\
\text { Afatinib, Osimertinib, Olmutinib, Nazartinib, Avitinib, lapatinib, Cetuximab, } \\
\text { Panitumumab, Crizotinib, Lenvatinib, Nilotinib, Pazopanib, Regorafenib, Sorafenib, } \\
\text { Sunitinib }\end{array}$ \\
\hline M (mesenchymal) & $\begin{array}{l}\text { Inhibit Scr, TGF } \beta \text {, EMT, Wnt, PI3K, } \\
\text { mTOR, IGFIR, Notch }\end{array}$ & $\begin{array}{l}\text { PI3K inhibitors Eg, Idelalisib } \\
\text { Scr inhibitors Eg, Bosutinib, Dasatinib } \\
\text { mTOR inhibitors Eg, Rapamycin, Everolimus, RapaLink-I } \\
\text { Growth Factor inhibitors Eg, Erlotinib, Gefitinib, Afatinib, Osimertinib, } \\
\text { Olmutinib, Nazartinib, Avitinib, lapatinib, Cetuximab, Panitumumab, Vandetanib, } \\
\text { Bevacizumab, Pertuzumab, Ramucirumab, Trastuzumab, Axitinib, Cabozantinib, } \\
\text { Ceritinib, Crizotinib, Lenvatinib, Nilotinib, Pazopanib, Regorafenib, Sorafenib, } \\
\text { Sunitinib }\end{array}$ \\
\hline $\begin{array}{l}\text { IM } \\
\text { (immunomodulatory) }\end{array}$ & Inhibit immune signaling & $\begin{array}{l}\text { Immune checkpoint inhibitors Eg, Ipilimumab, Nivolumab } \\
\text { PARP inhibitors Eg, Olaparib, Rucaparib, Talazoparib and Niraparib) } \\
\text { Cytostatics E.g.Cisplatin, Carboplatin, Nedaplatin, Eptaplatin, Oxaliplatin, } \\
\text { Lobaplatin, Satraplatin, Mercaptopurine }\end{array}$ \\
\hline BLI (basal-like I) & $\begin{array}{l}\text { Inhibit cell proliferation and DNA } \\
\text { damage response }\end{array}$ & $\begin{array}{l}\text { DNA Synthetic inhibitors Eg, Camptothecin, Doxorubicin, Daunorubicin, } \\
\text { Topotecan, Irinotecan, Mitomycin } \\
\text { PARP inhibitors Eg, Talazoparib, Niraparib, Olaparib, Rucaparib, } \\
\text { Cytostatics Eg, Oxaliplatin, Lobaplatin, Satraplatin, Cisplatin, Carboplatin, } \\
\text { Nedaplatin, Eptaplatin, Mercaptopurine } \\
\text { Mitosis inhibitors Eg, Ixabepilone, Nab-Paclitaxel, Vinorelbine Paclitaxel, } \\
\text { Docetaxel, }\end{array}$ \\
\hline BL2 (basal-like 2) & $\begin{array}{l}\text { Inhibit EGFR, TP63, and MET } \\
\text { signaling }\end{array}$ & $\begin{array}{l}\text { mTOR inhibitors Eg, RapaLink-I, everolimus, rapamycin, } \\
\text { PARP inhibitors Eg, Talazoparib, Rucaparib, Olaparib, and Niraparib } \\
\text { Cytostatics Eg, Oxaliplatin, Cisplatin, Nedaplatin, Carboplatin, Lobaplatin, } \\
\text { Satraplatin, Mercaptopurine, Eptaplatin, } \\
\text { Growth Factor inhibitors Eg Osimertinib, Gefitinib, Erlotinib, Bevacizumab, } \\
\text { Afatinib, Olmutinib, Nazartinib, Vandetanib, Avitinib, lapatinib, Ramucirumab, } \\
\text { Cetuximab, Panitumumab, Lenvatinib, Trastuzumab, Nilotinib, Pazopanib, } \\
\text { Regorafenib, Sorafenib, Sunitinib Pertuzumab, Axitinib, Cabozantinib, Ceritinib, } \\
\text { Crizotinib. }\end{array}$ \\
\hline
\end{tabular}

Note: Data from these studies ${ }^{42-44}$.

Abbreviations: PI3K, Phosphoinositide 3-kinases; FOXAI, Forkhead box protein AI (FOXAI), also known as hepatocyte nuclear factor 3-alpha (HNF-3A); AR, Androgen receptor; ERBB4, Receptor protein-tyrosine kinase ErbB-4; mTOR, mammalian target of rapamycin; EMT, epithelial-mesenchymal transition; Wnt, Wingless-related integration site; TGF $\beta$, Transforming growth factor beta; MAPK, mitogen-activated protein kinase; Rac, Ras-related C3 botulinum toxin substrate; PDGF, platelet-derived growth factor; IGFIR, insulin-like growth factor I; PARP, poly adenosine diphosphate-ribose polymerase. 
mitochondrial structural damage, and impairment of double-stranded DNA break repair in CSCs. CQ has the ability to effectively suppress TNBC cell proliferation in vitro and in a TNBC xenograft model. CQ reduces tumor development in carboplatin-resistant BRCA1 wildtype TNBC orthotopic xenografts by greatly eradicating the expression of DNA repair proteins in CSC populations when used in combination with other drugs. ${ }^{41}$ As a result, it is possible that CQ, an autophagy inhibitor with antiCSC effects, may be used as a TNBC treatment technique. However, it should be noted that no targeted therapy has received FDA approval for TNBC to date, and more reliable drug therapy against TNBC is urgently needed. The latest research aims to determine how natural metabolites and nano-enhanced natural metabolites can help combat triple-negative breast cancer.

Therapeutic strategies and targeted agents to treat TNBC are summarized in Table 1.

\section{Conclusion}

TNBC is an issue for patients and physicians since it has a poor prognosis and fewer treatment options, as seen by its high mortality rate when compared to other breast cancer subtypes. Even though TNBC is a serious disease, there is a solid foundation for its molecular subtypes that can cooccur in tumour. However, the underlying variability of the disease can limit the efficacy of treatment, despite molecular subtyping. Because of this, effective targeted remedies are becoming increasingly difficult to produce.

Some of the therapeutic approaches for TNBC management include targeting the DNA repair complex (platinum compounds and taxanes), p53 (taxanes), cell proliferation (anthracycline-containing regimen), and targeted therapy. The best adjuvant regimens for TNBC are still being developed. Several randomized trials have shown the benefits of adjuvant anthracyclines and taxanes in breast cancer.

Targeted treatments such as Poly (ADP-ribose) polymerase-1 (PARP-1), a nuclear DNA-binding enzyme triggered by DNA strand breaks, play a vital role in the signaling of DNA single-strand breaks as part of the repair process, are being developed successfully. This means that tailored therapeutics for TNBC must be developed immediately. Emerging treatment targets include tyrosine and non-tyrosine tyrosine kinases, PARP1, androgen receptor, immune-checkpoints, and epigenetic proteins discovered in recent molecular characterization of TNBCs.
Participating in a clinical trial of new treatments for TNBC, regardless of the stage of the disease, is a good option because TNBC is rare and has a high mortality rate (outcome) as compared to other types of breast cancer, and these trials provide patients with drugs that are not available in standard care.

\section{Abbreviations}

ARs, Androgen receptors; ERRs, Estrogen receptor dependent receptors; ERs, Estrogen receptors; HER2, Human epidermal growth factor receptor 2; MSL, Mesenchymal stem like; PRs, Progesterone receptors; TILs, Tumor infiltrating lymphocytes; TMAs, Tumor associated macrophages; TNBC, Triple Negative Breast Cancer.

\section{Acknowledgment}

The authors would like to acknowledge all the authors whose work has been reviewed in the preparation of the manuscript.

\section{Author Contributions}

All authors made a significant contribution to the work reported, whether that is in the conception, study design, execution, acquisition of data, analysis and interpretation, or in all these areas; took part in drafting, revising or critically reviewing the article; gave final approval of the version to be published; have agreed on the journal to which the article has been submitted; and agree to be accountable for all aspects of the work.

\section{Funding}

There is no funding to report.

\section{Disclosure}

The authors declare that they have no competing interests.

\section{References}

1. Polk A, Svane I-M, Andersson M, Nielsen D. Checkpoint inhibitors in breast cancer-current status. Cancer Treat Rev. 2018;63:122-134. doi:10.1016/j.ctrv.2017.12.008

2. Mitchell MP, Sharma P. The use of surgery and radiotherapy as treatment of regional nodes in breast cancer patients. Oncology. 2018;32(6): e52-e64.

3. Nounou MI, ElAmrawy F, Ahmed N, Abdelraouf K, Goda S, SyedSha-Qhattal H. Breast cancer: conventional diagnosis and treatment modalities and recent patents and technologies. Breast Cancer Basic Clin Res. 2015;9:BCBCR-S29420.

4. Arnedos M, Bihan C, Delaloge S, Andre F. Triple-negative breast cancer: are we making headway at least? Ther Adv Med Oncol. 2012;4(4):195-210. doi:10.1177/1758834012444711

5. Shang M, Chang C, Pei Y, Guan Y, Chang J, Li H. Potential management of circulating tumor DNA as a biomarker in triple-negative breast cancer. J Cancer. 2018;9(24):4627. doi:10.7150/jca.28458 
6. Lehmann BD, Bauer JA, Chen X, et al. Identification of human triple-negative breast cancer subtypes and preclinical models for selection of targeted therapies. $J$ Clin Invest. 2011;121 (7):2750-2767. doi:10.1172/JCI45014

7. Barton VN, D'Amato NC, Gordon MA, et al. Multiple molecular subtypes of triple-negative breast cancer critically rely on androgen receptor and respond to enzalutamide in vivo. Mol Cancer Ther. 2015;14(3):769-778. doi:10.1158/1535-7163.MCT-14-0926

8. Yan M, Li X, Tong D, et al. miR-136 suppresses tumor invasion and metastasis by targeting RASAL2 in triple-negative breast cancer. Oncol Rep. 2016;36(1):65-71. doi:10.3892/or.2016.4767

9. Wu Y-M, Chen Z-J, Jiang G-M, et al. Inverse agonist of estrogen-related receptor $\alpha$ suppresses the growth of triple negative breast cancer cells through ROS generation and interaction with multiple cell signaling pathways. Oncotarget. 2016;7(11):12568. doi:10.18632/oncotarget.7276

10. Maniscalco L, Millán Y, Iussich S, et al. Activation of mammalian target of rapamycin (mTOR) in triple negative feline mammary carcinomas. BMC Vet Res. 2013;9(1):1-9. doi:10.1186/1746-6148-9-80

11. Kim J-Y, Jung HH, Ahn S, et al. The relationship between nuclear factor (NF)- $\mathrm{B}$ family gene expression and prognosis in triple-negative breast cancer (TNBC) patients receiving adjuvant doxorubicin treatment. Sci Rep. 2016;6(1):1-11. doi:10.1038/s41598-016-0001-8

12. Huang L, Liu Z, Chen S, Liu Y, Shao Z. A prognostic model for triple-negative breast cancer patients based on node status, cathepsin-D and Ki-67 index. PLoS One. 2013;8(12):e83081. doi:10.1371/journal.pone.0083081

13. Chae BJ, Bae JS, Lee A, et al. p53 as a specific prognostic factor in triple-negative breast cancer. Jpn J Clin Oncol. 2009;39(4):217-224. doi:10.1093/jjco/hyp007

14. Rampurwala M, Wisinski KB, O'Regan R. Role of the androgen receptor in triple-negative breast cancer. Clin Adv Hematol Oncol. 2016;14(3):186.

15. Bonnefoi H, Grellety T, Tredan O, et al. A Phase II trial of Abiraterone acetate plus prednisone in patients with triple-negative androgen receptor positive locally advanced or metastatic breast cancer (UCBG 12-1). Ann Oncol. 2016;27(5):812-818. doi:10.1093/ annonc/mdw067

16. Traina TA, Miller K, Yardley DA, et al. Results from a Phase 2 study of enzalutamide (ENZA), an androgen receptor (AR) inhibitor, in advanced AR+ triple-negative breast cancer (TNBC). Am Soc Clin Oncol. 2015. doi:10.1200/jco.2015.33.15_suppl.1003

17. Parker JS, Peterson AC, Tudor IC, Hoffman J, Uppal H. A novel biomarker to predict sensitivity to enzalutamide (ENZA) in TNBC. Am Soc Clin Oncol. 2015. doi:10.1200/jco.2015.33.15_suppl.1083

18. Mina A, Yoder R, Sharma P. Targeting the androgen receptor in triple-negative breast cancer: current perspectives. Onco Targets Ther. 2017;10:4675. doi:10.2147/OTT.S126051

19. Lehmann BD, Jovanović B, Chen X, et al. Refinement of triple-negative breast cancer molecular subtypes: implications for neoadjuvant chemotherapy selection. PLoS One. 2016;11(6): e0157368. doi:10.1371/journal.pone.0157368

20. Prat A, Adamo B, Cheang MC, Anders CK, Carey LA, Perou CM. Molecular characterization of basal-like and non-basal-like triple-negative breast cancer. Oncologist. 2013;18(2):123. doi:10.1634/theoncologist.2012-0397

21. Ring BZ, Hout DR, Morris SW, et al. Generation of an algorithm based on minimal gene sets to clinically subtype triple negative breast cancer patients. BMC Cancer. 2016;16(1):1-8.

22. Zhu A, Li Y, Song W, et al. Antiproliferative effect of androgen receptor inhibition in mesenchymal stem-like triple-negative breast cancer. Cell Physiol Biochem. 2016;38(3):1003-1014. doi:10.1159/ 000443052

23. Caiazza F, Murray A, Madden SF, et al. Preclinical evaluation of the AR inhibitor enzalutamide in triple-negative breast cancer cells. Endocr Relat Cancer. 2016;23(4):323-334. doi:10.1530/ERC-16-0068
24. Pardoll DM. The blockade of immune checkpoints in cancer immunotherapy. Nat Rev Cancer. 2012;12(4):252-264. doi:10.1038/ nrc3239

25. García-Teijido P, Cabal ML, Fernández IP, Pérez YF. Tumorinfiltrating lymphocytes in triple negative breast cancer: the future of immune targeting. Clin Med Insights Oncol. 2016;10:CMOS34540.

26. Wang J, Chen H, Chen X, Lin H. Expression of tumor-related macrophages and cytokines after surgery of triple-negative breast cancer patients and its implications. Med Sci Mon. 2016;22:115. doi:10.12659/MSM.895386

27. Qiao Y, He H, Jonsson P, Sinha I, Zhao C, Dahlman-Wright K. AP-1 is a key regulator of proinflammatory cytokine $\mathrm{TNF} \alpha$-mediated triple-negative breast cancer progression. J Biol Chem. 2016;291 (10):5068-5079. doi:10.1074/jbc.M115.702571

28. Browne G, Dragon JA, Hong D, et al. MicroRNA-378-mediated suppression of Runx1 alleviates the aggressive phenotype of triple-negative MDA-MB-231 human breast cancer cells. Tumor Biol. 2016;37(7):8825-8839. doi:10.1007/s13277-015-4710-6

29. Adams BD, Wali VB, Cheng CJ, et al. miR-34a silences c-SRC to attenuate tumor growth in triple-negative breast cancer. Cancer Res. 2016;76(4):927-939. doi:10.1158/0008-5472.CAN-15-2321

30. Manning BD, Cantley LC. AKT/PKB signaling: navigating downstream. Cell. 2007;129(7):1261-1274. doi:10.1016/j.cell.2007. 06.009

31. Chen Y-J, Yeh M-H, Yu M-C, et al. Lapatinib-induced NF-kappaB activation sensitizes triple-negative breast cancer cells to proteasome inhibitors. Breast Cancer Res. 2013;15(6):1-14. doi:10.1186/bcr3575

32. Rubio-Viqueira B, Hidalgo M. Targeting mTOR for cancer treatment. Adv Exp Med Biol. 2006;587:309-327.

33. Pikarsky E, Porat RM, Stein I, et al. NF- $\kappa$ B functions as a tumour promoter in inflammation-associated cancer. Nature. 2004;431 (7007):461-466. doi:10.1038/nature02924

34. Wang C-Y, Mayo MW, Baldwin AS. TNF-and cancer therapyinduced apoptosis: potentiation by inhibition of NF-кB. Science. 1996;274(5288):784-787. doi:10.1126/science.274.5288.784

35. Phan B, Majid S, Ursu S, et al. Tumor suppressor role of microRNA-1296 in triple-negative breast cancer. Oncotarget. 2016;7(15):19519. doi:10.18632/oncotarget.6961

36. Zhang H, Cohen AL, Krishnakumar S, et al. Patient-derived xenografts of triple-negative breast cancer reproduce molecular features of patient tumors and respond to mTOR inhibition. Breast Cancer Res. 2014;16(2):1-16. doi:10.1186/bcr3640

37. Singh JC, Novik Y, Stein S, et al. Phase 2 trial of everolimus and carboplatin combination in patients with triple negative metastatic breast cancer. Breast Cancer Res. 2014;16(2):1-8. doi:10.1186/ bcr3634

38. Moestue SA, Dam CG, Gorad SS, et al. Metabolic biomarkers for response to PI3K inhibition in basal-like breast cancer. Breast Cancer Res. 2013;15(1):1-18. doi:10.1186/bcr3391

39. Ellard SL, Clemons M, Gelmon KA, et al. Randomized phase II study comparing two schedules of everolimus in patients with recurrent/metastatic breast cancer: NCIC Clinical Trials Group IND. 163. Clin Oncol. 2009;27(27):4536-4541.

40. Hart S, Novotny-Diermayr V, Goh KC, et al. VS-5584, a novel and highly selective PI3K/mTOR kinase inhibitor for the treatment of cancer. Mol Cancer Ther. 2013;12(2):151-161. doi:10.1158/15357163.MCT-12-0466

41. Liang DH, Choi DS, Ensor JE, Kaipparettu BA, Bass BL, Chang JC. The autophagy inhibitor chloroquine targets cancer stem cells in triple negative breast cancer by inducing mitochondrial damage and impairing DNA break repair. Cancer Lett. 2016;376(2):249-258. doi:10.1016/j.canlet.2016.04.002

42. Oualla K, El-Zawahry HM, Arun B, et al. Novel therapeutic strategies in the treatment of triple-negative breast cancer. Ther Adv Med Oncol. 2017;9(7):493-511. doi:10.1177/1758834017711380 
43. Lehmann BD, Pietenpol JA. Identification and use of biomarkers in treatment strategies for triple-negative breast cancer subtypes. J Pathol. 2014;232(2):142-150. doi:10.1002/path.4280
44. Yao H, He G, Yan S, et al. Triple-negative breast cancer: is there a treatment on the horizon? Oncotarget. 2017;8(1):1913. doi:10.18 632/oncotarget. 12284

\section{Publish your work in this journal}

Breast Cancer - Targets and Therapy is an international, peer-reviewed open access journal focusing on breast cancer research, identification of therapeutic targets and the optimal use of preventative and integrated treatment interventions to achieve improved outcomes, enhanced survival and quality of life for the cancer patient.
The manuscript management system is completely online and includes a very quick and fair peer-review system, which is all easy to use. Visit $\mathrm{http}: / / \mathrm{www}$. dovepress.com/testimonials.php to read real quotes from published authors. 